\title{
Programa Mais Médicos: contexto de implantação e efeito no provimento de médicos na atenção primária à saúde no Brasill, 2008 a 2016
}

\author{
Elzo Pereira Pinto Junior, ${ }^{1}$ Leila Denise Alves Ferreira Amorim ${ }^{2}$ e Rosana Aquino ${ }^{3}$
}

Como citar

Pinto Junior EP, Amorim LDAF, Aquino R. Programa Mais Médicos: contexto de implantação e efeito no provimento de médicos na atenção primária à saúde no Brasil, 2008 a 2016. Rev Panam Salud Publica. 2020;44:e23. https://doi.org/10.26633 /RPSP.2020.23

RESUMO

Objetivo. Caracterizar os municípios brasileiros que compunham os perfis prioritários para implantação do Programa Mais Médicos (PMM) e avaliar o efeito do programa no provimento emergencial de médicos na atenção primária à saúde (APS) no Brasil segundo contexto de implantação.

Métodos. Estudo de séries temporais interrompidas, que considerou como desfecho as taxas de médicos de APS/10 000 habitantes no Brasil e nos contextos de implantação do PMM. Para essa análise de série temporal, foram coletados dados mensais de janeiro de 2008 a dezembro de 2016. O início da intervenção foi estabelecido no mês de julho de 2013, data da assinatura da medida provisória que criou o PMM. Os municípios foram caracterizados segundo aspectos demográficos, socioeconômicos, serviços de saúde e distribuição de médicos.

Resultados. A criação do PMM resultou em incremento na taxa de médicos de APS em todos os perfis prioritários para a implantação do programa, com destaque para municípios com mais de 20\% dos habitantes vivendo em situação de pobreza. Além disso, o estudo demonstrou diferenças marcantes entre os municípios brasileiros em aspectos socioeconômicos, na organização de serviços de saúde e na disponibilidade de médicos na APS, inclusive dentro de um mesmo contexto de implantação do PMM.

Conclusões. O PMM tem contribuído para reduzir a escassez e a má distribuição de médicos na APS no Brasil, reduzindo as inequidades de acesso. Desse modo, foi bem-sucedido em seu eixo de provimento emergencial, tornando-se uma importante estratégia de fortalecimento da APS e do Sistema Único de Saúde.

Palavras-chave Recursos humanos; distribuição de médicos; atenção primária à saúde; Estratégia Saúde da Família; Sistema Único de Saúde; Brasil.

A escassez e a má distribuição de médicos são problemas comuns em diversos países no mundo (1), mesmo naqueles considerados de alta renda e com sistemas de saúde universal, como o Reino Unido (2) e o Canadá (3). Especificamente no âmbito dos serviços de atenção primária à saúde (APS), a insuficiência de médicos (4) põe em risco a universalidade do acesso e a continuidade do cuidado nesse nível de atenção, considerado a principal porta de entrada e o ordenador da assistência em outros níveis dos sistemas de saúde (5).

No Brasil, o eixo organizacional da APS é a Estratégia Saúde da Família (ESF), um modelo que atua em prevenção, promoção e reabilitação por meio de equipes multiprofissionais, comprometidas com a integralidade da assistência. Esse modelo é focado na família e guiado pelo perfil epidemiológico

\footnotetext{
Universidade Federal da Bahia (UFBA), Departamento de Estatística, Salvador (BA), Brasil.

3 Universidade Federal da Bahia (UFBA), Instituto de Saúde Coletiva (ISC), Programa de Pós-Graduação em Saúde Coletiva, Salvador (BA), Brasil.
}

Fundação Oswaldo Cruz (Fiocruz), Centro de Integração de Dados e Conhecimentos para Saúde (CIDACS), Salvador (BA), Brasil. $\square$ elzojr@hot mail.com 
e sociocultural da comunidade (6). A ESF funciona a partir de equipes que devem contar, minimamente, com um médico, um enfermeiro, um técnico de enfermagem e de quatro a seis agentes comunitários de saúde.

Desde sua criação, em meados da década de 1990, a ESF tem expandido sua cobertura populacional especialmente nos municípios mais pobres, sendo que essa expansão tem sido associada à melhoria das condições de saúde da população brasileira $(7,8)$. Apesar desses avanços, a carência de médicos na APS ainda é um desafio para o Sistema Único de Saúde (SUS), comprometendo a qualidade e a integralidade da atenção (9).

Para tentar solucionar a escassez de médicos e garantir o provimento desses profissionais em áreas remotas, diversas estratégias foram implementadas no âmbito internacional: políticas educacionais, com ampliação de vagas de graduação e pós-graduação; políticas de regulação que envolvem a instituição de serviços obrigatórios em áreas carentes e a abertura de vagas em municípios mais pobres; incentivos monetários, que incluem aditivos nos salários dos médicos; incentivos nãomonetários, como a extensão de vistos de permanência para médicos estrangeiros (4).

Nesse contexto, em 2013, o governo brasileiro criou o Programa Mais Médicos (PMM) (10). O PMM tem como principais finalidades reduzir a carência de médicos em regiões consideradas prioritárias para o SUS, diminuir as inequidades na distribuição desses profissionais no território brasileiro, melhorar a prestação de serviços no âmbito da APS, aprimorar a formação médica e ampliar a inserção dos médicos no sistema público de saúde (10).

Os documentos normativos que orientam o PMM definiram regiões prioritárias para a implantação desse programa no âmbito do SUS, enfatizando áreas remotas, de extrema pobreza e com outras situações de vulnerabilidade. Os grupos de municípios considerados elegíveis foram aqueles com $20 \%$ ou mais da população vivendo em extrema pobreza, o grupo de 100 municípios mais pobres do país (G100), as capitais, as regiões metropolitanas e os demais municípios (1). Com o PMM, um grande contingente de médicos foi alocado aos municípios que aderiram ao programa, especialmente na região Nordeste (1). Em 2015, foram contabilizadas 15820 equipes de ESF com médicos do PMM em todo o Brasil, o que representava a presença de médicos em 70\% dos municípios (11).

Nesse contexto, os objetivos deste trabalho foram caracterizar os municípios brasileiros que compunham os perfis prioritários para implantação do PMM e avaliar o efeito do programa no provimento emergencial de médicos na APS no Brasil segundo contexto de implantação.

\section{MATERIAIS E MÉTODOS}

A avaliação do efeito do PMM no provimento de médicos em serviços de APS utilizou a metodologia de séries temporais interrompidas (interrupted time-series analysis ou ITSA). Nessa metodologia, com alto grau de validade interna $(12,13)$, múltiplas observações de um desfecho de interesse são analisadas antes e depois de uma dada intervenção. Este estudo considerou como desfecho a taxa de médicos de APS por 10000 habitantes.

Foram coletados dados mensais, de janeiro de 2008 a dezembro de 2016, de 5665 municípios brasileiros, sendo considerado como início da intervenção o mês de julho de 2013, data da assinatura da medida provisória que criou o PMM.
Foram excluídos cinco municípios criados após 2010, por não possuírem informações importantes para a descrição sociodemográfica, econômica e de serviços de saúde.

As informações sobre o quantitativo de médicos foram obtidas a partir do Cadastro Nacional de Estabelecimentos de Saúde (CNES), consultado via site do Departamento de Informática do SUS (DATASUS). A definição da variável "médicos de serviços de APS" seguiu três critérios, sendo o primeiro estar registrado em um dos seguintes códigos da Classificação Brasileira de Ocupações, versão 2002: 225125 - médico clínico; 225130 - médico de família e comunidade; 225142 - médico da ESF; 225170 - médico generalista. O segundo critério foi prestar atendimentos nos serviços do SUS; e o terceiro foi exercer seu trabalho em posto de saúde, centro de saúde/unidade básica, policlínica, unidade mista, unidade móvel fluvial ou unidade móvel terrestre.

Para a construção da variável taxa de médicos de APS, utilizou-se como numerador o número de médicos de serviços de APS, conforme descrição acima, e como denominador a população total do município em 2015, multiplicada por 10000. Esse indicador foi calculado para cada um dos perfis de municípios do PMM: capitais, regiões metropolitanas, G100, 20\% de pobreza e outros municípios. Definiu-se como "município com PMM" aquele que, em dezembro de 2015, tinha pelo menos um médico do programa.

Além das variáveis relacionadas à oferta de médicos nos serviços de APS, foram utilizadas informações sociodemográficas, econômicas e de serviços de saúde dos municípios, a fim de obter a descrição de suas características. Para isso, foram consultados dados secundários do Sistema de Informações sobre Nascidos Vivos (SINASC), Sistema de Informações da Atenção Básica (SIAB), Instituto Brasileiro de Geografia e Estatística (IBGE) e Ministério do Desenvolvimento Social e Combate à Fome (MDS).

A caracterização dos municípios se deu a partir dos seguintes blocos: primeiro, situação socioeconômica, composta por indicadores do IBGE, sendo a população residente estimada para o ano de 2015; e demais indicadores do censo de 2010, taxa de urbanização, expectativa de vida ao nascer, renda per capita e proporção de analfabetos com 15 anos ou mais. O segundo bloco englobou características dos serviços de saúde, inclusive indicadores referentes ao ano de 2015 de cobertura populacional da ESF, cobertura de atenção pré-natal (7 ou mais consultas), consultas médicas por ESF/habitantes, leitos hospitalares/1 000 habitantes e leitos hospitalares do SUS/1 000 habitantes. Esses indicadores foram obtidos a partir dos dados do SIAB, do SINASC e do CNES. O terceiro bloco incluiu indicadores da disponibilidade de médicos, obtidos para o ano 2015: número de médicos do PMM; número de médicos do PMM/10 000 habitantes, número de médicos não PMM na ESF/10 000 habitantes, número de médicos na ESF/10 000 habitantes e proporção de médicos do PMM na ESF (\%).

$\mathrm{Na}$ descrição dos municípios brasileiros, foram calculadas as medianas de cada indicador. Para comparar a distribuição dessas medidas, de acordo com a adesão ao PMM (aderiu/não aderiu) em cada um dos contextos de implantação, aplicou-se o teste estatístico de Mann-Whitney, admitindo-se diferenças estatisticamente significativas quando o valor de $P<0,05$.

Para a avaliação do efeito do PMM na taxa de médicos, foram estimados coeficientes obtidos a partir de modelos de séries temporais interrompidas para múltiplos grupos, quando 
é possível a construção de um grupo intervenção (com o PMM) e de um grupo controle (sem o PMM). No caso específico das capitais, por não existir nenhuma capital no país que não estivesse participando do PMM, foi realizada a análise de séries temporais interrompidas para um único grupo (14).

A estimação usando ITSA foi feita através de mínimos quadrados generalizados em modelos de regressão lineares usando ajuste para autocorrelação serial (15). No caso da ITSA para um único grupo, o modelo incluiu um termo produto entre o indicador de intervenção e o indicador de período (antes ou depois da intervenção). Assume-se ainda que qualquer variável confundidora tempo-dependente está mudando muito lentamente, de modo que seria distinguível do efeito acentuado do indicador de intervenção. Essa suposição é mais plausível se não são observadas tendências em período anterior à intervenção. No caso de ITSA com múltiplos grupos, assume-se que variáveis confundidoras não observadas ou omitidas afetam de forma semelhante os grupos de comparação.

A análise foi conduzida no software Stata, versão 15, tendo sido utilizado o pacote ITSA (14), que fornece erros-padrão Newey-West para lidar com autocorrelação e possível heterocedasticidade (variância constante dos resíduos gerados pela estimação do modelo), e que assume que os erros aleatórios do modelo seguem um processo autorregressivo de ordem 1 (AR1). Para a construção dos modelos, optou-se por utilizar a opção "prais" do Stata com o intuito de estimar os parâmetros da regressão controlados pela autocorrelação de primeira ordem, dada a dependência dos dados.

Também foram construídas medidas adicionais de pós-estimação para enriquecer as análises de séries temporais interrompidas, conforme proposto anteriormente (16), utilizando-se os seguintes parâmetros: i. tendência pré-intervenção: controle; ii. tendência pré-intervenção: tratamento; iii. diferença pré-intervenção: tratamento versus controle; iv. tendência pós-intervenção: controle; v. tendência pós-intervenção: tratamento; vi. diferença pós-intervenção: tratamento versus controle; vii. diferença pré-intervenção versus pós-intervenção: controle; viii. diferença pré-intervenção versus pós-intervenção: tratamento; ix. diferença pré-intervenção versus pós-intervenção: tratamento versus controle.

A interpretação dos parâmetros de tendência linear (i, ii, iv, v) considerou tendências de aumento nas taxas quando o coeficiente é positivo e o valor de $P<0,05$; tendências de redução das taxas quando o coeficiente é negativo e o valor de $P<0,05$; e tendência estacionária quando $P>0,05$. Analogamente, para a análise das diferenças de tendências nos períodos antes e depois da intervenção e nos grupos intervenção e controle (parâmetros iii, vi, vii, viii, ix) foram considerados como estatisticamente significativos os resultados cujo valor de $P<0,05$.

Em relação às questões éticas, por se tratar de um estudo com dados secundários de acesso público, sem possibilidade de identificação dos sujeitos, este estudo não necessitou de aprovação por comitê de ética em pesquisa.

\section{RESULTADOS}

A descrição dos grupos de municípios do PMM evidenciou que todas as capitais possuíam médicos vinculados ao programa. As capitais apresentaram as maiores medianas para tamanho da população (1 013643 habitantes), taxa de urbanização $(99,5 \%)$, renda per capita (R\$ 1 052,00), número de leitos hospitalares (3,3/1 000 habitantes), leitos hospitalares do SUS (2,1/1 000 habitantes) e número total de médicos do PMM (51 médicos). Apesar desses indicadores positivos, destacam-se os baixos valores nas medianas da cobertura da ESF $(47,3 \%)$ (tabela 1).

Dos 508 municípios que compunham as regiões metropolitanas, 76,4\% aderiram ao PMM. A comparação das características socioeconômicas dos municípios com e sem PMM mostrou que aqueles que aderiram ao programa apresentavam maior população $(P<0,001)$, maior taxa de urbanização $(P<0,001)$ e menor proporção de analfabetos entre os indivíduos com 15 anos ou mais $(P=0,003)$. Em relação aos aspectos organizacionais dos serviços de saúde, não foram observadas diferenças estatisticamente significativas entre os dois tipos de municípios (tabela 1).

No G100, apenas cinco municípios não aderiram ao PMM. Não foram observadas diferenças estatisticamente significativas quanto às características sociodemográficas e de organização dos serviços de saúde nos municípios com e sem o PMM (tabela 1).

Dos 1652 municípios classificados no grupo com $20 \%$ da população em situação de extrema pobreza, 1314 (79,5\%) aderiram ao PMM. Foram observadas diferenças estatisticamente significativas em um conjunto de indicadores sociodemográficos, tendo os municípios que aderiram ao programa apresentado maior número de habitantes $(P<0,001)$, maior esperança de vida ao nascer $(P=0,002)$ e menor proporção de analfabetos com 15 anos ou mais (valor de $P<0,001$ ). Em relação aos serviços de saúde, os municípios de ambos os grupos apresentaram mediana de $100 \%$ na cobertura da ESF, mas os demais indicadores relacionados aos serviços de saúde foram melhores naqueles que não aderiram ao PMM (tabela 1).

O grupo dos municípios que não se enquadravam em nenhuma dessas classificações, categorizados em "outros municípios", incluiu 3280 cidades, das quais 2802 (63,5\%) aderiram ao PMM. A análise da situação socioeconômica e demográfica revelou que os municípios com PMM apresentaram maior porte populacional (valor de $P<0,001$ ). Por outro lado, os municípios que não aderiram ao $\mathrm{PMM}$ possuíam maior expectativa de vida ao nascer (valor de $P=0,001$ ). Tais achados indicam que os municípios que aderiram ao PMM tinhas piores indicadores sociais, demográficos e econômicos. A análise dos indicadores relacionados aos serviços de saúde também evidenciou que os melhores resultados, em todos os quesitos analisados, foram observados no grupo de municípios que não aderiram ao PMM (tabela 1).

\section{Efeito do PMM no provimento de médicos nos serviços de APS}

No período que antecedeu a criação do PMM, as taxas de médicos de APS eram menores no grupo de municípios que posteriormente aderiram ao programa, tanto em nível nacional quanto nos diferentes contextos de implantação. Após a criação do PMM, as taxas de médicos aumentaram em todos os grupos de municípios, especialmente naqueles que aderiram ao PMM. A variação da taxa de médicos de APS foi de 1,56 para 2,11 no grupo que aderiu ao programa, enquanto nos demais municípios foi de 2,02 para 2,14, considerando junho de 2013 e dezembro de 2016, respectivamente. Em alguns desses cenários, como nos municípios com $20 \%$ ou mais da população em extrema pobreza, o grupo que aderiu ao PMM alcançou, em 


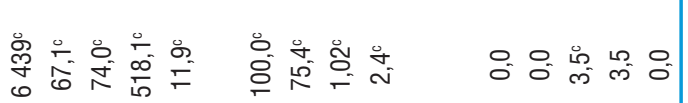

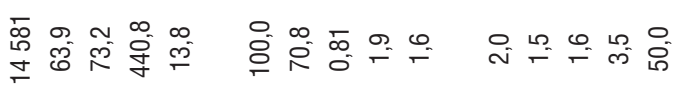

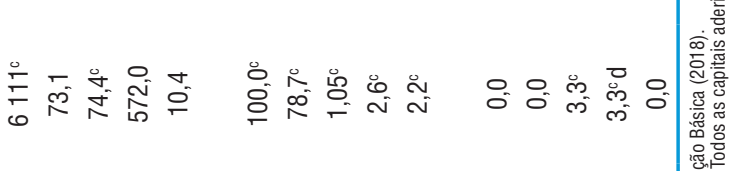

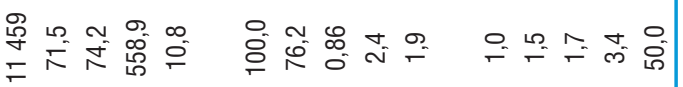



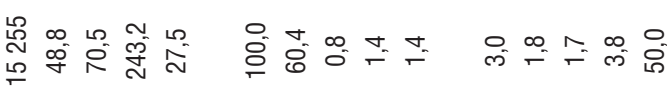

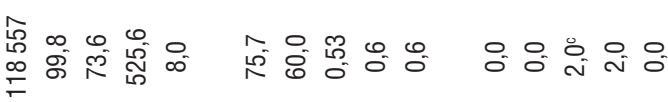

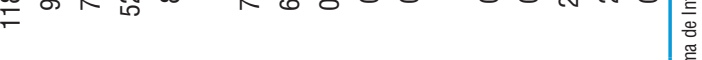

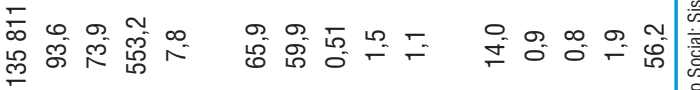

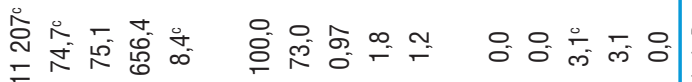



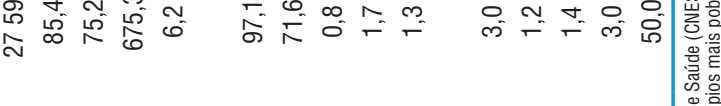

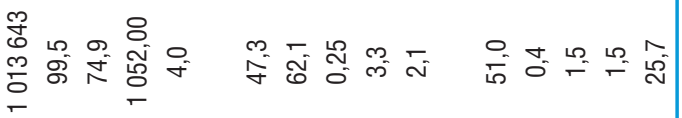

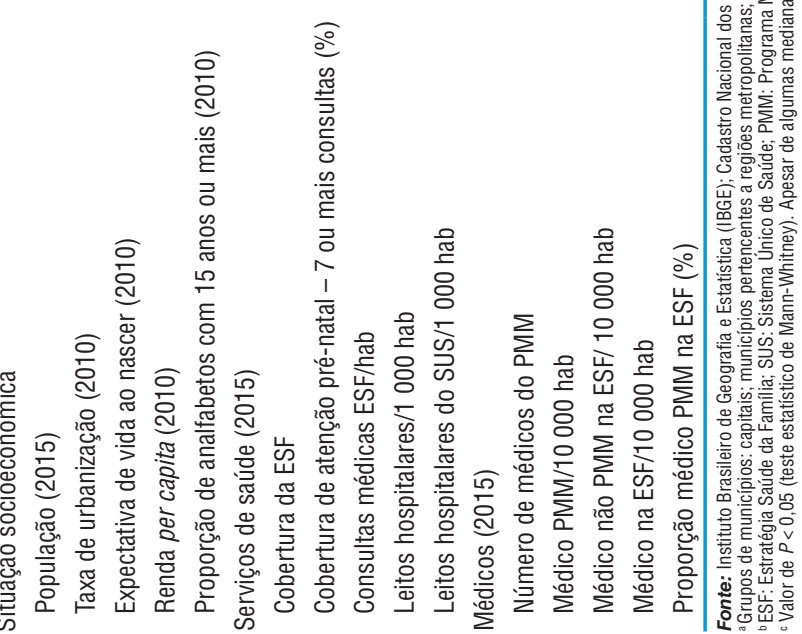


TABELA 2. Taxas de médicos na atenção primária à saúde nos municípios brasileiros segundo contextos de implantação (grupos de municípios) e adesão ao Programa Mais Médicos, 2008 a 2016

\begin{tabular}{lccc}
$\begin{array}{c}\text { Grupos de municípios/ } \\
\text { adesão ao Programa } \\
\text { Mais Médicosa }\end{array}$ & \multicolumn{3}{c}{$\begin{array}{c}\text { Taxa de médicos na atenção primária } \\
\text { à saúde/10 000 habitantes }\end{array}$} \\
\cline { 2 - 4 } $\begin{array}{c}\text { Capital } \\
\text { Sim }\end{array}$ & 1,71 & 1,49 & 1,83 \\
Jegeiro/2008 & Junho/2013 & Dezembro/2016 \\
Região metropolitana & & & \\
$\quad$ Não & 2,40 & 2,24 & 2,29 \\
Sim & 1,83 & 1,65 & 2,06 \\
20\% pobreza & & & \\
Não & 2,38 & 2,24 & 2,22 \\
Sim & 1,77 & 1,63 & 2,51 \\
G100 & & & \\
Não & 1,97 & 2,02 & 2,33 \\
Sim & 1,45 & 1,33 & 1,73 \\
Outros & & & \\
Não & 2,16 & 1,92 & 2,07 \\
Sim & 1,80 & 1,61 & 2,33 \\
Brasil & & & \\
Não & 2,23 & 2,02 & 2,14 \\
Sim & 1,74 & 1,56 & 2,11 \\
\hline
\end{tabular}

Fonte: Cadastro Nacional dos Estabelecimentos de Saúde (2018).

aGrupos de municípios: capitais; municípios pertencentes a regiões metropolitanas; $\mathrm{G} 100=100$ municípios mais pobres do país; $20 \%$ pobreza = municípios com $20 \%$ ou mais da população vivendo em extrema pobreza; outros = demais municípios. dezembro de 2016, maior taxa de médicos de APS do que aqueles que não aderiram ao programa (tabela 2).

As taxas de médicos de APS apresentavam tendência estacionária nos municípios brasileiros antes da criação do PMM. No entanto, após sua implantação, houve aumento dessas taxas no grupo de municípios que aderiu ao programa e tendência estacionária naqueles que não aderiram. Assim, foram observadas diferenças estatisticamente significativas nas taxas de médicos de APS entre os grupos com e sem intervenção no período seguinte à criação do PMM. Esse comportamento também foi observado nas análises que envolveram o grupo de municípios classificados como "outros" (tabela 3 e figura 1).

Nos municípios classificados como "G100" e "regiões metropolitanas" foi possível observar tendência estacionária para a taxa de médicos de APS nos grupos com e sem PMM, antes de julho de 2013. Após essa data, a tendência pós-intervenção evidenciou aumento na taxa de médicos de APS no grupo que aderiu ao programa. Por sua vez, no grupo que não aderiu, esse indicador permaneceu sem alteração significativa (tabela 3 e figura 1).

A tendência das taxas de médicos de APS nos municípios com $20 \%$ ou mais da população vivendo em extrema pobreza apresentou tendência estacionária no período anterior à criação do PMM. Esse comportamento estacionário se manteve no período pós-intervenção nos municípios que não aderiram ao PMM. Nos municípios que participavam do programa, foi observada tendência de aumento na oferta de médicos nos serviços de APS (tabela 3 e figura 1 ).

TABELA 3. Impacto do Programa Mais Médicos nas tendências das taxas de médicos na atenção primária à saúde nos municípios brasileiros segundo contextos de implantação (grupos de municípios)a , 2008 a 2016

\begin{tabular}{|c|c|c|c|c|c|c|c|c|c|c|}
\hline \multirow{2}{*}{$\begin{array}{l}\text { Taxa de médicos na atenção } \\
\text { primária à saúde }\end{array}$} & \multicolumn{2}{|c|}{ Brasil } & \multicolumn{2}{|c|}{ G100 } & \multicolumn{2}{|c|}{$20 \%$ pobreza } & \multicolumn{2}{|c|}{ Regiões metropolitanas } & \multicolumn{2}{|c|}{ Outros } \\
\hline & Coeficiente & $P$-valor & Coeficiente & $P$-valor & Coeficiente & $P$-valor & Coeficiente & $P$-valor & Coeficiente & $P$-valor \\
\hline \multicolumn{11}{|l|}{$\begin{array}{l}\text { Metodologia de séries temporais } \\
\text { interrompidas - múltiplos grupos }\end{array}$} \\
\hline 1. Tendência pré-intervenção: controle & $-0,0027$ & 0,121 & 0,0020 & 0,355 & $-0,0020$ & 0,526 & $-0,0020$ & 0,277 & $-0,0034$ & 0,153 \\
\hline 2. Tendência pré-intervenção: tratamento & $-0,0018$ & 0,299 & $-0,0009$ & 0,678 & 0,0000 & 0,994 & $-0,0014$ & 0,433 & $-0,0020$ & 0,394 \\
\hline $\begin{array}{l}\text { 3. Diferença pré-intervenção: tratamento } \\
\text { versus controle }\end{array}$ & 0,0009 & 0,715 & $-0,0030$ & 0,344 & 0,0019 & 0,658 & 0,0006 & 0,830 & 0,0014 & 0,683 \\
\hline 4. Tendência pós-intervenção: controle & 0,0033 & 0,196 & 0,0018 & 0,629 & $-0,0014$ & 0,761 & 0,0042 & 0,132 & 0,0040 & 0,222 \\
\hline 5. Tendência pós-intervenção: tratamento & 0,0134 & $0,000^{\mathrm{b}}$ & 0,0098 & $0,007^{b}$ & 0,0212 & $0,000^{\mathrm{b}}$ & 0,0104 & $0,000^{\mathrm{b}}$ & 0,0175 & $0,000^{\mathrm{b}}$ \\
\hline $\begin{array}{l}\text { 6. Diferença pós-intervenção: tratamento } \\
\text { versus controle }\end{array}$ & 0,0101 & $0,005^{b}$ & 0,0081 & 0,117 & 0,0225 & $0,001^{b}$ & 0,0062 & 0,116 & 0,0135 & $0,004^{b}$ \\
\hline $\begin{array}{l}\text { 7. Diferença pré-intervenção versus } \\
\text { pós-intervenção: controle }\end{array}$ & 0,0060 & 0,096 & $-0,0003$ & 0,955 & 0,0006 & 0,924 & 0,0062 & 0,120 & 0,0069 & 0,792 \\
\hline $\begin{array}{l}\text { 8. Diferença pré-intervenção versus } \\
\text { pós-intervenção: tratamento }\end{array}$ & 0,0152 & $0,000^{\mathrm{b}}$ & 0,0108 & $0,034^{b}$ & 0,0212 & $0,001^{b}$ & 0,0119 & $0,003^{b}$ & 0,0195 & $0,000^{\mathrm{b}}$ \\
\hline $\begin{array}{l}\text { 9. Diferença pré-intervenção versus } \\
\text { pós-intervenção: tratamento versus } \\
\text { controle }\end{array}$ & 0,0051 & 0,072 & 0,0110 & 0,122 & 0,0206 & $0,023^{b}$ & 0,0057 & 0,314 & 0,0121 & 0,066 \\
\hline \multicolumn{11}{|l|}{$\begin{array}{l}\text { Metodologia de séries temporais } \\
\text { interrompidas - grupo único - } \\
\text { capitais }\end{array}$} \\
\hline 1. Tendência pré-intervenção & $-0,0033$ & $0,028^{b}$ & & & & & & & & \\
\hline 2. Tendência pós-intervenção & 0,0072 & $0,003^{b}$ & & & & & & & & \\
\hline $\begin{array}{l}\text { 3. Diferença da tendência pré- } \\
\text { intervenção versus pós-intervenção }\end{array}$ & 0,0105 & $0,002^{b}$ & & & & & & & & \\
\hline
\end{tabular}


FIGURA 1. Tendência das taxas de médicos na atenção primária à saúde/10 000 habitantes segundo perfil de município e adesão ao Programa Mais Médicos, Brasil, 2008 a 2016
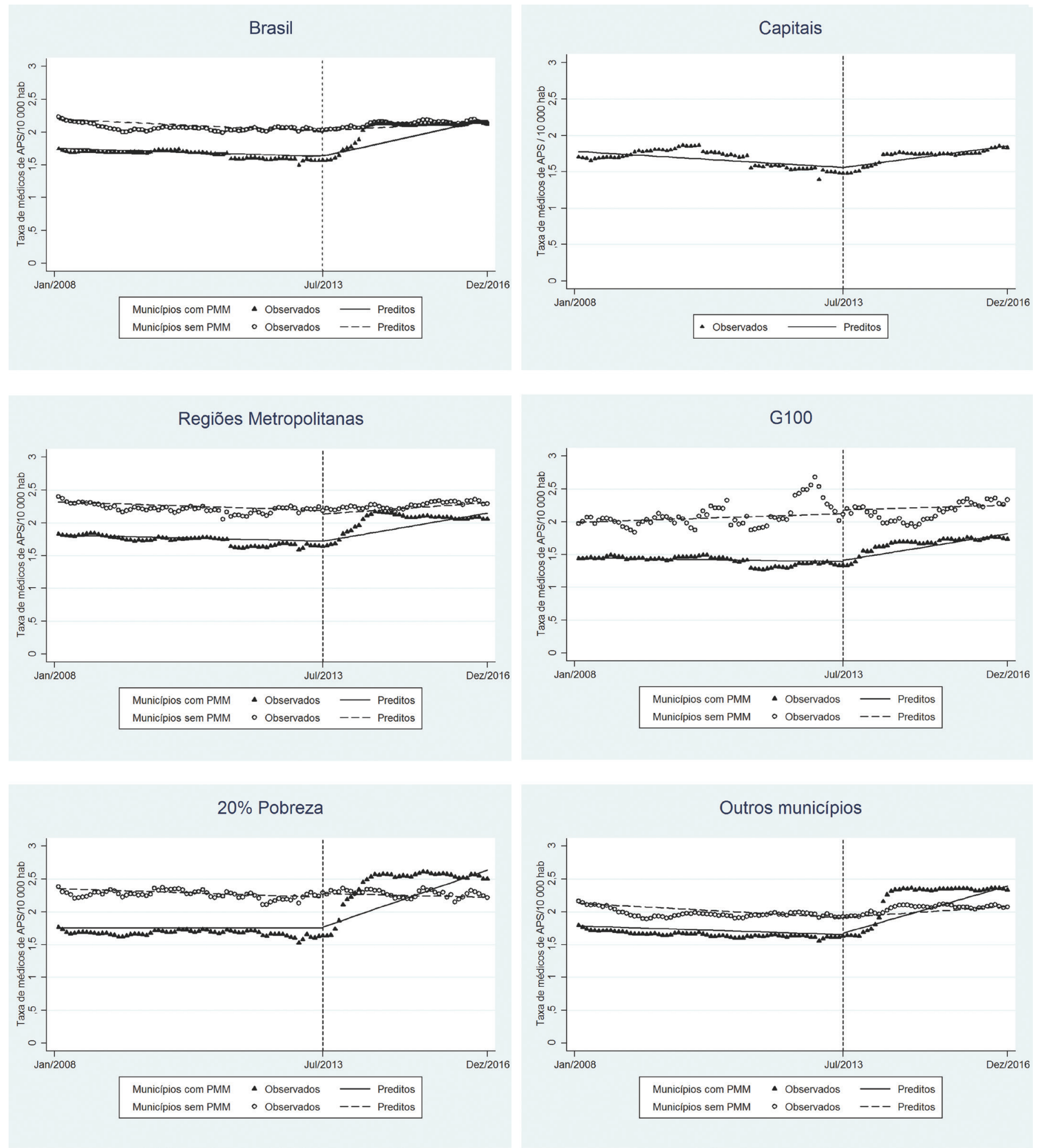

Fonte: Cadastro Nacional dos Estabelecimentos de Saúde (2018).

${ }^{a}$ Grupos de municípios: capitais; municípios pertencentes a regiões metropolitanas; $G 100=100$ municípios mais pobres do país; $20 \%$ pobreza = municípios com $20 \%$ ou mais da população vivendo em extrema pobreza; outros = demais municípios. Início do PMM: julho de 2013. Regressão de Prais-Winsten e Cochrane-Orcutt- lag(1). 
Nas capitais dos estados e no Distrito Federal houve tendência de redução na taxa de médicos de APS antes da criação do PMM. Esse comportamento foi modificado com a criação do PMM, tendo esses municípios experimentado uma tendência de crescimento nessas taxas após a implementação do programa (tabela 3 e figura 1 ).

\section{DISCUSSÃO}

Os resultados deste estudo evidenciaram que a criação do PMM foi acompanhada de um significativo aumento na taxa de médicos de APS em todos os perfis prioritários para a implantação do programa, o que comprova o seu sucesso no eixo provimento emergencial. Apesar de o aumento no provimento de médicos ter sido observado em todo o território nacional, vale destacar que em municípios com maior vulnerabilidade, como aqueles que possuíam mais de $20 \%$ de sua população em situação de extrema pobreza, o efeito foi ainda mais substancial.

Os primeiros resultados demonstram que, de 2013 a 2014, 14168 médicos foram destinados aos municípios que aderiram ao PMM, sendo a região Nordeste aquela que recebeu o maior quantitativo de médicos no período (4 853) (1). Ao final de 2015, foi possível observar que, com o PMM, todas as unidades da federação apresentavam crescimento no número de médicos; esse crescimento foi menor que $10 \%$ em apenas cinco estados (17).

Além de evidenciar os efeitos do PMM no provimento de médicos, esta pesquisa também demonstrou que os municípios brasileiros apresentam grande diversidade em aspectos socioeconômicos, na organização de seus serviços de saúde e na disponibilidade de médicos na APS. Isso fica ainda mais evidente quando se constata que municípios classificados num mesmo contexto de implantação apresentaram diferenças importantes nos seus indicadores. Esses achados indicam a importância de realizar análises de implantação e de efeito do PMM segundo contextos específicos.

Os municípios brasileiros que aderiram ao PMM apresentavam, inicialmente, piores indicadores socioeconômicos e de serviços de saúde. Contudo, após a criação desse programa, já contavam com taxas de médicos na ESF semelhantes às dos municípios com melhores condições socioeconômicas e que não haviam aderido ao programa. Por se tratar de uma iniciativa de provimento emergencial que buscou alocar médicos em cenários de maior escassez, esse achado se coaduna com o objetivo do eixo de provimento emergencial, conforme a lei que rege o PMM.

Num estudo sobre o efeito do PMM no provimento desses profissionais em áreas remotas e vulneráveis do país, que englobou principalmente municípios das regiões Norte e Nordeste, foi observada melhoria na densidade de médicos e redução do número de municípios que não apresentavam nenhum médico (18). Além de pesquisas empíricas, estudos de revisão de literatura têm evidenciado o impacto do PMM no provimento desses profissionais no Brasil $(19,20)$.

A má distribuição de médicos no Brasil pode ser explicada, entre outros, por aspectos socioeconômicos dos municípios, como produto interno bruto (PIB), índice de desenvolvimento humano (IDH) e condições de violência e acesso a bens e serviços. Esse conjunto de características se expressa em desigualdades em diversos níveis geográficos, envolvendo marcantes diferenças entre ambientes rurais e urbanos, municípios do interior ou da capital e zonas centrais ou periféricas de grandes cidades (21).

$\mathrm{O}$ presente estudo não analisou o perfil dos médicos que foram alocados nos municípios que aderiram ao PMM. Ainda assim, é importante ressaltar que, além dos médicos brasileiros, formados no Brasil ou no exterior, o programa contou com a participação de médicos de mais de 40 países, com destaque para os médicos cubanos (22). Nesse contexto, evidências têm apontado que aspectos relacionados ao processo de trabalho dos médicos estrangeiros, especialmente os cubanos, como a cultura da prevenção e a humanização no atendimento, foram avaliados positivamente por usuários e gestores (23).

A participação de médicos estrangeiros nos serviços de APS no Brasil foi um desafio enfrentado pelo governo brasileiro para garantir o sucesso do eixo provimento emergencial do PMM. A convocação desses profissionais estrangeiros ocorreu após o lançamento do primeiro edital de chamamento, que contou com a adesão de milhares de municípios, gerando uma demanda que não foi preenchida por médicos brasileiros. $\mathrm{O}$ processo gerou tensões decorrentes dos interesses defendidos pelas entidades médicas e da responsabilidade pública de garantir o acesso a serviços de saúde no âmbito da APS que contassem com equipes completas, o que inclui a presença do médico, a fim de se ofertar cuidado resolutivo e de qualidade (24).

Apesar de muitas evidências apontarem melhorias nas condições de saúde da população brasileira a partir do PMM, o programa foi descontinuado em dezembro de 2018, com importante descontinuidade na oferta de profissionais médicos nos serviços de APS, o que pode comprometer as condições de saúde da população (25).

Em relação às limitações deste estudo, aponta-se a utilização de um único ponto no tempo para descrever as características dos municípios, de modo que seria interessante que futuras investigações comparassem essas características no período anterior e posterior à criação do PMM. Além disso, a indisponibilidade de dados de acesso público sobre as características socioeconômicas e de serviços de saúde dos Distritos Sanitários Especiais Indígenas (DSEI) impossibilitaram a expansão das análises para esse importante contexto de implantação. Apesar dessas limitações, é preciso destacar a importância de construir descrições mais detalhadas das características dos municípios e das tendências das taxas de médicos por habitante, segundo os contextos de implantação e a adesão ao PMM. A utilização dessa estratégia permitiu capturar sutis diferenças nas características e nas tendências que passariam despercebidas em análises de agregados maiores.

Outro elemento a ser destacado foi o uso de STIA para avaliar o efeito do PMM no provimento. A STIA é um desenho indicado para avaliação do efeito de intervenções em larga escala cuja implantação tenha se dado em um período bem definido. Outra vantagem é que a STIA pode ser aplicada em estudos cuja intervenção não foi alocada de modo randomizado. Nesse sentido, permite a comparação de indicadores observados após a intervenção com valores preditos a partir da tendência apresentada por tais indicadores no momento anterior à implantação da intervenção (26). A aplicabilidade da STIA tem se destacado na avaliação de um conjunto de intervenções, sejam elas políticas públicas, leis ou campanhas, em desfechos de interesse para a saúde pública (27).

Os resultados desta pesquisa demonstraram que os municípios brasileiros apresentam grande diversidade em aspectos 
sociodemográficos, na organização dos serviços de saúde e na disponibilidade de médicos nos serviços de APS. Além disso, este estudo evidenciou que o PMM contribuiu para reduzir a escassez e a má distribuição de médicos na APS no Brasil. Nesse sentido, o eixo de provimento emergencial do PMM se desenvolveu com sucesso e se tornou uma importante estratégia de fortalecimento da APS e do SUS. Os achados desta pesquisa, ao demonstrar os efeitos positivos de um programa de provimento emergencial de médicos, podem contribuir para que outros países utilizem estratégias semelhantes àquelas adotadas no Brasil, como definição de municípios prioritários e convocação de médicos estrangeiros, na tentativa de solucionar os problemas de escassez e má distribuição de médicos nos serviços de APS.

Contribuições dos autores. EPPJ concebeu a ideia original, planejou o estudo e coletou e analisou os dados. LDAFA planejou o estudo, analisou os dados e interpretou os resultados. RA concebeu a ideia original, planejou o estudo, analisou os dados e interpretou os resultados. Todos os autores participaram da redação do manuscrito e aprovaram a versão final.

Conflitos de interesse. Nada declarado pelos autores.

Financiamento. EPPJ recebeu bolsa de doutorado da Coordenação de Aperfeiçoamento de Pessoal de Nível Superior (CAPES) e do Conselho Nacional de Desenvolvimento Científico e Tecnológico (CNPq).

Declaração. As opiniões expressas no manuscrito são de responsabilidade exclusiva dos autores e não refletem necessariamente a opinião ou política da RPSP/PAJPH ou da Organização Pan-Americana da Saúde (OPAS).

\section{REFERÊNCIAS}

1. Oliveira JPA, Sanchez MN, Santos LMP. O Programa Mais Médicos: provimento de médicos em municípios brasileiros prioritários entre 2013 e 2014. Cienc Saude Colet. 2016;21(9):2719-27.

2. Asaria M, Cookson R, Fleetcroft R, Ali S. Unequal socioeconomic distribution of the primary care workforce: whole-population small area longitudinal study. BMJ Open. 2016;6(1): e008783.

3. Guttmann A, Shipman SA, Lam K, Goodman DC, Stukel TA. Primary care physician supply and children's health care use, access, and outcomes: findings from Canada. Pediatrics. 2010;125(6):1119-26.

4. Alves SMC, de Oliveira FP, Matos MFM, Santos LMP, Delduque MC. Cooperação internacional e escassez de médicos: análise da interação entre Brasil, Angola e Cuba. Cienc Saude Colet. 2017;22(7): 2223-35.

5. Starfield B. Atenção primária: equilíbrio entre necessidades de saúde, serviços e tecnologia. Brasília: UNESCO/Ministério da Saúde; 2002. Disponível em: https://www.nescon.medicina.ufmg .br/biblioteca/imagem/0253.pdf Acessado em junho de 2019.

6. Fertonani HP, de Pires DEP, Biff D, Scherer MDA. Modelo assistencial em saúde: conceitos e desafios para a atenção básica brasileira. Cienc Saude Colet. 2015;20(6):1869-78.

7. Macinko J, Harris MJ. Brazil's family health strategy — delivering community-based primary care in a universal health system. N Engl J Med. 2015;372(23):2177-81.

8. Paim J, Travassos C, Almeida C, Bahia L, Macinko J. The Brazilian health system: history, advances, and challenges. Lancet. 2011;377(9779):1778-97.

9. Santos LMP, Costa AM, Girardi SN. Programa Mais Médicos: uma ação efetiva para reduzir iniquidades em saúde. Cienc Saude Colet. 2015;20(11):3547-52.

10. Brasil. Lei 12 871/2013. Brasília: Diário Oficial da União; 2013. Disponível em: http://www.planalto.gov.br/ccivil_03/_ato2011 -2014/2013/Lei/L12871.htm Acessado em junho de 2018.

11. Miranda GMD, Mendes ACG, da Silva ALA, dos Santos Neto PM. A ampliação das equipes de saúde da família e o programa mais médicos nos municípios brasileiros. Trab Educ Saude. 2017;15(1):131-45.

12. Campbell DT, Stanley JC. Experimental and quasi-experimental designs for research. $1^{\mathrm{a}}$ ed. Chicago, IL: Rand McNally; 1963.

13. Shadish WR, Cook TD, Campbell DT. Experimental and quasiexperimental designs for generalized causal inference. $2^{\mathrm{a}}$ ed. Boston: Cengage Learning; 2001.

14. Linden A. Conducting interrupted time-series analysis for singleand multiple-group comparisons. Stata J. 2015;15(2):480-500.

15. Box GEP, Jenkins GM, Reinsel GC. Time series analysis: forecasting and control. $1^{\text {a }}$ ed. São Francisco: Holden-Day; 1976.

16. Linden A. A comprehensive set of postestimation measures to enrich interrupted time-series analysis. Stata J. 2017;17(1):73-88.
17. Pinto HA, de Oliveira FP, Santana JSS, Santos FOS, de Araujo SQ, de Figueiredo AM, et al. The Brazilian more doctors program: evaluating the implementation of the provision axis from 2013 to 2015. Interface Comun Saude Educ. 2017;21(1 Suppl):1087-101.

18. Santos LMP, Oliveira A, Trindade JS, Barreto ICHC, Palmeira PA, Comes $Y$, et al. Implementation research: towards universal health coverage with more doctors in Brazil. Bull World Health Organ. 2017;95(2):103-12.

19. Kemper ES, Mendonça AVM, de Sousa MF. Programa Mais Médicos: panorama da produção científica. Cienc Saude Colet. 2016;21(9):2785-96.

20. Mourão JJ, Rodrigues ARM, Aragão OC, Goyanna NF, Cavalcante AES, Vasconcelos MAS, et al. Programa Mais Médicos e suas contribuições para a saúde no Brasil: revisão integrativa. Rev Panam Salud Publica. 2018;42(1):e2.

21. de Oliveira APC, Gabriel M, Poz MRD, Dussault G. Desafios para assegurar a disponibilidade e acessibilidade à assistência médica no sistema único de saúde. Cienc Saude Colet. 2017;22(4):1165-80.

22. Kemper ES, Tasca R, Harzheim E, Jiménez JMS, Hadad J, Sousa MF. Cobertura universal em saúde e o Programa Mais Médicos no Brasil. Rev Panam Salud Publica. 2018;42(1):e1.

23. Santos JBF, Maciel RHMO, Lessa MGG, Maia ALLN, Guimarães EPA. Médicos estrangeiros no Brasil: a arte do saber olhar, escutar e tocar. Saude Soc. 2016;25(4):1003-16.

24. Alessio MM, de Sousa MF. Programa mais médicos: elementos de tensão entre governo e entidades médicas. Interface. 2017;21(1 Suppl):1143-56.

25. Santos LMP, Millet C, Rasella D, Hone T. The end of Brazil's More Doctors programme? BMJ. 2018;363(1):k5247.

26. Bernal JL, Cummins S, Gasparrini A. Interrupted time series regression for the evaluation of public health interventions: a tutorial. Int J Epidemiol. 2017;46(1):348-55.

27. Kontopantelis E, Doran T, Springate DA, Buchan I, Reeves D. Regression based quasi-experimental approach when randomisation is not an option: interrupted time series analysis. BMJ. 2015;350(1):h2750.

Manuscrito recebido em 26 de dezembro de 2018. Aceito em versão revisada em 22 de maio de 2019. 


\section{More Doctors Program: context of implementation and effect on the provision of primary health care physicians in Brazil, 2008-2016}

ABSTRACT Objective. To characterize the Brazilian municipalities in each More Doctors Program (MDP) priority profile and to assess the effect of the program on the emergency supply of primary care physicians in Brazil according to implementation context.

Method. An interrupted time-series analysis (ITSA) was performed, considering as outcome the rate of primary care physicians/10 000 inhabitants in Brazil and within each MDP implementation context. Monthly data were collected from January 2008 to December 2016. The intervention was considered to have started in July 2013 , when the MDP was officially created. The municipalities were characterized according to demographic, socioeconomic, health care service, and physician distribution variables.

Results. Establishment of the MDP was associated with increased rate of primary care physicians in all priority profiles, especially in municipalities in which at least $20 \%$ of the population lived in poverty. Furthermore, the study showed significant differences among Brazilian municipalities in socioeconomic aspects, health care service organization, and availability of primary care physicians, even within the same MDP implementation context.

Conclusion. The MDP has contributed to reduce the shortage and improve the distribution of primary care physicians in Brazil, thus reducing inequalities in access to services. Consequently, the MDP was successful in its emergency supply of physicians, having become an important strategy to strengthen primary health care and the Brazilian Unified Health System.

Keywords Workforce; physicians distribution; primary health dare; Family Health Strategy; Unified Health System; Brazil.

\section{Programa Mais Médicos: Contexto de implementación y efecto en la dotación de médicos de atención primaria de salud en Brasil,, 2008-2016}

RESUMEN

Palabras clave
Objetivo. Caracterizar los municipios brasileños representativos de los perfiles prioritarios para la implementación del programa Mais Médicos (PMM) y evaluar el efecto del programa en la dotación de médicos de atención primaria de salud (APS) en situaciones de emergencia en Brasil, según el contexto de implementación. Métodos. Se realizó un análisis de series cronológicas interrumpidas, en el cual se consideraron como resultado las tasas de dotación de médicos de APS por cada 10000 habitantes en Brasil y en el contexto de implementación del PMM. Para dicho análisis, se recolectaron datos mensuales desde enero del 2008 hasta diciembre del 2016. Se determinó que la intervención comenzó en el mes de julio del 2013, fecha de la firma de la medida provisional en virtud de la cual se creó el PMM. Los municipios se caracterizaron según sus características demográficas y socioeconómicas, los servicios de salud y la distribución de los médicos en cada uno.

Resultados. La creación del PMM redundó en un incremento de la tasa de dotación de médicos de APS en todos los perfiles prioritarios para la implementación del programa, con hincapié en los municipios donde más de $20 \%$ de los habitantes se encontraban en situación de pobreza. Además, el análisis demostró que había notables diferencias entre los municipios brasileños en cuanto al aspecto socioeconómico, a la organización de los servicios de salud y a la disponibilidad de médicos de APS, incluso dentro del mismo contexto de implementación del PMM.

Conclusión. El PMM ha contribuido a reducir la escasez y la mala distribución de los médicos de APS en Brasil, con lo cual ha disminuido la inequidad del acceso a los servicios. En ese sentido, tuvo éxito en su eje de dotación de médicos en situaciones de emergencia, hecho que lo convirtió en una importante estrategia de fortalecimiento de la APS y del Sistema Único de Salud.

Recursos humanos; distribución de médicos; atención primaria de salud; Estrategia de Salud Familiar; Sistema Único de Salud; Brasil. 